\title{
Mood, Cognitive Function and Quality of Life Improvements in Middle Aged Women Following Supplementation with Polygonum minus Extract (Penambahbaikan Mood dan Kualiti Kehidupan dalam Kalangan Wanita Pertengahan Umur Selepas Pemberian Ekstrak Polygonum minus)
}

\author{
HANIS MASTURA YAHYA*, SUZANA SHAHAR, SITI NUR ARINA ISMAIL, AINOR FARAHIN AZIZ, \\ NORMAH CHE DIN \& BIBI NABIHAH ABDUL HAKIM
}

\begin{abstract}
Polygonum minus is a plant rich in flavonoids and antioxidants beneficial for reducing oxidative stress and lipid peroxidation in neuronal membranes. This randomized, double-blind, placebo-controlled study evaluated the potential benefits of P. minus extract (LineMinus ${ }^{T M}$ ) towards improving cognitive function, mood status and quality of life. Thirty five middle-aged women (35-55 years old) were randomized into intervention $(\mathrm{n}=17)$ and control group $(\mathrm{n}=18)$. Two capsules of P. minus (250 mg) or placebo (100 mg maltodextrin) each were taken once daily for six weeks. Cognitive tests, mood and anthropometric measurements were measured at baseline, week 3 and week 6, whilst biomarkers were measured at baseline and week 6. Parameters related to mood and quality of life including energy/fatigue, social functioning and general health significantly improved from baseline to week 6 in the intervention group $(\mathrm{p}<0.05)$. Mean score for cognitive tests (i.e. digit span, comprehensive trail making test (CTMT) and three domains of CNS vital sign (CNSVS)] improved significantly in both intervention and control groups $(\mathrm{p}<0.05)$. There was a significant decrease of mean uric acid, estimated glomerular filtration rate (eGFR), total cholesterol and glycated hemoglobin (HbAlC) in the intervention group from baseline to week 6. P. minus supplementation has the potential to improve mood and quality of life and no adverse effects were reported by the participants after 6 weeks supplementation.
\end{abstract}

Keywords: Flavonoid; mood; Polygonum minus; phytochemicals; quality of life; supplement

\section{ABSTRAK}

Polygonum minus adalah tumbuhan yang kaya dengan flavonoid dan antioksidan serta bermanfaat untuk mengurangkan tekanan oksidatif dan peroksidasi lipid pada membran neuron. Kajian ini berbentuk rawak, dwi-buta dan plasebo terkawal untuk menilai potensi faedah ekstrak P. minus (LineMinus ${ }^{T M}$ ) dalam meningkatkan fungsi kognitif, status mood dan kualiti hidup. Tiga puluh lima wanita pertengahan umur (berumur 35-55 tahun) dibahagikan secara rawak ke dalam kumpulan intervensi $(\mathrm{n}=17)$ dan kumpulan kawalan $(\mathrm{n}=18)$. Dua kapsul $\mathrm{P}$. minus (250 mg) atau plasebo (100 mg maltodekstrin) telah diambil setiap sehari selama enam minggu. Ujian kognitif, mood dan beberapa ukuran antropometri telah diukur pada peringkat dasar, minggu 3 dan minggu 6, manakala penanda biologi diukur pada peringkat dasar dan minggu 6. Parameter yang berkaitan dengan mood dan kualiti hidup termasuk tenaga/keletihan, fungsi sosial dan kesihatan meningkat secara signifikan pada peringkat dasar hingga minggu 6 untuk kumpulan intervensi $(\mathrm{p}<0.05)$. Min skor untuk ujian kognitif seperti Digit Span, Comprehensive Trail Making Test (CTMT) dan tiga domain CNS Vital Sign (CNSVS)] meningkat secara signifikan dalam kedua-dua kumpulan intervensi dan kawalan (p<0.05). Terdapat penurunan yang signifikan dalam min asid urik, anggaran kadar penapisan glomerular (EGFR), jumlah kolesterol dan hemoglobin glycated (HbAlC) dalam kumpulan intervensi pada peringkat dasar dan minggu 6. Pemberian P. minus mempunyai potensi untuk memperbaik mood dan kualiti hidup serta tiada kesan buruk dilaporkan oleh peserta selepas pengambilan selama 6 minggu.

Kata kunci: Fitokimia; flavonoid; kualiti hidup; mood; Polygonum minus; suplemen

\section{INTRODUCTION}

Globally, there is an increase in the number of individuals with mental disorders related to neurological, behavioural and substances usage. The number has been predicted to increase up to $15 \%$ in the year 2020 (WHO 2004). There has been growing interest in the use of complementary and alternative medicines as a natural method for treating or preventing numerous mental disorders and improving cognitive function and mood. Several traditional herbs including passion flower, valerian root and kava have been utilized for centuries as remedies to assist in mind calmness and mood enhancement (Lakhan \& Viera 2010). Furthermore, numerous studies have attempted to identify the efficacy and safety of utilizing alternative medicines in treating psychological problems specifically mental health such as anxiety disorder (Garcia-Garcia et al. 
2008; Kinrys et al. 2009; Saeed et al. 2007). The usage of herbal and other natural remedies for the management and treatment of psychological conditions is timely due to the rising cost of prescription medications and their potential unwanted side effects to the patients.

Herbs may improve cognitive function or reduce the risk of cognitive decline. For example, supplementation of American ginseng (Panax quinquefolius) was reported to increase the working memory performance up to $10 \%$ among young adults in Australia (Scholey et al. 2010). A modest improvement was found in the accuracy of working memory task after 14 day administration of Ginkgo biloba extract among middle-aged adults (Silberstein et al. 2011). In addition, supplementation of SuperUlam, a mixture of herbs containing about $14.5 \%$ Polygonum minus as well as other ingredients such as sireh extract, pegaga extract, turmeric extract, curry leaves extract and selasih extract have shown to improve cognitive function among healthy individual aged 35-65 years old (Udani 2013).

P. minus is an aromatic plant originates from Southeast Asia, belonging to the family Polygonaceae and locally known as kesum. It has been used as a traditional remedy and for cooking purpose to provide flavour and fragrance. Previous study has reported that $P$. minus extract showed the highest antioxidant activity as compared to other popular herbs such as 'pegaga' and curry leaves (Huda-Faujan et al. 2009). P. minus is usually consumed as salad or 'ulam' (a Malaysian practice of complementing raw plants with rice dishes) (Al-Qari 2006; Cheah et al. 2008; Joseph et al. 2005; Sumazian et al. 2010; Tanaka 1976). Traditional usage of $P$. minus leaves decoctions includes treatment for dandruff, diarrhoea and indigestion (Herbal Medicine Research Centre 2002; Norliza et al. 2013). Nutrients found in P. minus include carotenes, retinol equivalents and L-ascorbic acid, $\alpha$-tocopherol (vitamin E), calcium, phosphorus, iron, sodium, potassium, magnesium, copper and zinc (Ching \& Mohamed 2001). It has been proven to be a potent natural source of antioxidant due to high antioxidant activity among other selected Malaysian plants (Azlim Almey et al. 2010; Qader et al. 2011). There were numerous compounds previously isolated from $P$. minus leaves including flavonoids namely myricetin, quercetin, isorhamnetin, catechin and rutin (Narasimhulu \& Mohamed 2014; Qader et al. 2012).

Until recently, reports on the effect of $P$. minus on mood and quality of life are still limited. There is a need to explore the potential effect of P.minus on cognition and mood status since we have previously shown that 6 weeks supplementation of $P$. minus had improved short term memory and attention among subjects with poor mood and improved IQ among those with good mood (Suzana et al. 2015). Therefore, this study aimed to evaluate the benefits of $P$. minus supplementation towards mood and cognitive function among middle aged women.

\section{MATERIALS AND METHODS}

\section{STUDY DESIGN}

This study was a randomised, double-blind, placebocontrolled trial assessing the effect of $P$. minus extract (LineMinusTM) on cognition and mood status among middle aged women in Klang Valley, Malaysia. Subjects were randomised into two groups, namely placebo or $P$. minus. Randomisation was conducted using random sample cases decided by statistical computer programme, Statistical package for Social Sciences version (SPSS). Blinding procedure is ensured by labelling the $P$. minus and placebo capsules as either A or B Only so that the manufacturer knew the coding for both A and B labelled capsules.

\section{DATA COLLECTION}

A total of 63 subjects were recruited from the screening process which involved self-administered questionnaires, anthropometric and blood pressure measurement and a collection of $20 \mathrm{~mL}$ fasting venous blood after 8 to $10 \mathrm{~h}$ fasting for the analysis of $\mathrm{HbA} 1 \mathrm{C}$, serum lipid, renal profile and liver function. The inclusion criteria include women aged 35 to 55 years with body mass index (BMI) of less than $40.0 \mathrm{~kg} / \mathrm{m}^{2}$. The exclusion criteria included individuals with or had a history of substance or alcohol abuse, history of major depression (marked as depressed mood most of the day) and bipolar disorder, had medical conditions problems (e.g. uncontrolled diabetes and kidney problem), pregnancy or lactation. A total of 35 subjects participated in the study and were randomly assigned into either placebo or intervention group. This study has obtained approval from the Medical Research Ethics Committee of the Universiti Kebangsaan Malaysia (NN-2014-060) and subjects have given their informed consent in accordance with the principles in the declaration of Helsinki (World Medical Association declaration of Helsinki, 2000) and Good Clinical Practice Guidelines.

\section{INTERVENTION}

This study was performed over a 6 week intervention period which includes three visits within the study period. Data collection was conducted from October until December 2014 at a few schools and a government office in Klang Valley in Malaysia. Each subject was randomly chosen where 17 subjects were selected from schools and the other 18 subjects were from government office. These subjects were evaluated for outcome measures including a cognitive function test battery, mood test and quality of life at three time points (baseline, week 3 and week 6). Blood profiles including $\mathrm{HbA1C}$, serum lipid, renal profile, liver function and blood pressure were analyzed at baseline and week 6 to measure the effect of $P$. minus supplementation on health status. Subjects were advised to restrict their intake of caffeinated drinks no more than two cups daily on the testing days and to abstain the intake of any vitamins, other herbal supplements during the study period. 
The subjects were provided with two bottles of supplement each containing 60 capsules throughout the intervention period. The treatment group received capsules containing P. minus extract whilst the controlled group received placebo capsules containing $100 \mathrm{mg}$ maltodextrin. The placebo used was a sensory-identical capsule. Both groups were required to take two capsules once daily preferably in the morning. Subjects were required to record the time of consumption each day and noted down the reason for not taking the products if any in a diary provided to them for compliance checking. Both diary and capsules were brought back to the study site during scheduled visits and daily reminder was performed through phone call or short message service (SMS). Compliance of the subjects was monitored regularly whereby about $98.3 \%$ of the given capsules were consumed by the subjects.

\section{STUDY PRODUCT}

The supplements capsule, LineMinusTM was a standardized water extract of $P$. minus leaves with a ratio of water to dried leaves of P. minus (1:20) (George et al. 2014; Ming et al. 2013). A finished product contained 250 $\mathrm{mg}$ of $P$. minus extract for each besides containing about $0.4 \%$ and $0.1 \%$ Quercetin-3-glucuronide and Quercitrin, respectively. Table 1 shows the constituents of both placebo and $P$. minus. This product has passed microbial limit test with absence of several microbes in the products and were reported to be free from Salmonella, Escherichia coli and Staphylococcus aureus. The level of several heavy metals such as arsenic, cadmium, lead and mercury were analyzed and passed within the regulated limits. Reference from previous study that stated no-observed-adverse-effect level (NOAEL) of P. minus extract in Wistar rats following oral administration for 28 days at more than $1000 \mathrm{mg} / \mathrm{kg}$ body weight was used to determine the dosage of two capsules (i.e. $500 \mathrm{mg} / \mathrm{d}$ ) (Ming et al. 2013). According to the manufacturers' guidelines, this supplement only can be used traditionally for general health and not recommended for pregnancy and lactation women due to lack of sufficient data.

\section{OUTCOME MEASURES}

Cognitive function, mood and quality of life are the primary outcome of this study. These variables were measured through Digit Span (Groth-Marnat 2000), Rey Auditory verbal learning test (RAVLT) (Rey 1964), comprehensive trail making test (CTMT) (Reynolds 2002), Wechsler abbreviated scale of intelligence (WASI) (Wechsler 1999) and CNS vital sign (CNSVS) (Gualtieri \& Johnson 2006), profile of mood states (POMS) (McNair et al. 1971) and 36Item short form health survey (SF-36) (Brazier et al. 1992). Anthropometry and body composition measurements were the secondary outcomes and measured at baseline, week 3 and week 6 . The anthropometric parameters were measured with minimal clothing using TANITA digital lithium scale HD3 19 to the nearest $0.1 \mathrm{~kg}$, (TANITA Corporation, Japan) for body weight and Leicester Portable Height Measure (SECA, German) for height. A total of $20 \mathrm{~mL}$ of fasting blood samples were taken for analysis of fasting serum lipid, liver and renal function test (triglycerides, total cholesterol, low-density lipoprotein cholesterol, highdensity lipoprotein cholesterol) and $\mathrm{HbA} 1 \mathrm{C}$ were measured at baseline and 6 weeks. Blood pressure measurement was performed using an automated monitor (Omron HEM 7321-E, Netherlands). At each visit, subjects were asked by research assistants for any symptoms or side effects that they experienced.

TABLE 1. Energy, nutrient, and bioactive profile of P. minus preparation, LineMinusTM and placebo capsules

\begin{tabular}{|c|c|c|}
\hline Constituents (per $100 \mathrm{~g}$ ) & Placebo & P. minus preparation, LineMinusTM \\
\hline \multicolumn{3}{|l|}{ Energy $^{1}$} \\
\hline$(\mathrm{kJ})$ & 386 & 305 \\
\hline (kcal) & 1621 & 1281 \\
\hline \multicolumn{3}{|l|}{ Macronutrients ${ }^{1}$} \\
\hline Fat $(\mathrm{g})$ & 0 & 0 \\
\hline Carbohydrate $(\mathrm{g})$ & 94 & 67.6 \\
\hline Protein $(g)$ & 2.6 & 8.6 \\
\hline \multicolumn{3}{|l|}{ Micronutrients 2,3} \\
\hline Calcium (mg) & 6.3 & 38.5 \\
\hline Iron (mg) & 0.4 & 1.4 \\
\hline Vitamin A $(\mu \mathrm{g})$ & 0 & 0 \\
\hline L-ascorbic acid (mg) & 10.6 & 27.0 \\
\hline$\alpha$-tocopherol (mg) & 0 & 0 \\
\hline \multicolumn{3}{|l|}{ Bioactive content $(\%)^{3}$} \\
\hline Quercetin-3-glucuronide & - & 0.4 \\
\hline Quercitrin & - & 0.1 \\
\hline
\end{tabular}

${ }^{1}$ Determined by Method of Analysis for Nutritional Labeling, AOAC 1993

${ }^{2}$ Determined by U.S Environmental Protection Agency Method (EPA) revision 2, 1995

${ }^{3}$ Determined by High Performance Liquid Chromatography (HPLC) 


\section{STATISTICAL ANALYSIS}

Data was analysed using Statistical package for Social Sciences version (SPSS) 20 (v 20.0; IBM Corporation, Armonk, NY, USA). Data normality was determined from the Shapiro-Wilk test. The results were stated as either mean \pm standard deviation (SD), frequency or percentage or with $95 \%$ confidence interval. The variations between the treatment and the placebo groups were analysed using independent student t-test and chi squared test. Paired sample t-tests were used for within subjects means comparisons between control and intervention groups at baseline, Week 3 and Week 6. All tests were 2-tailed with $\mathrm{p}$ values less than 0.05 were considered statistically significant.

\section{RESULTS}

A total of 63 subjects were screened, with 43 were eligible and consented to participate. However, 35 were included in the final analysis, as four had health problems that interfered with the protocol of the study and one subject was lost to follow up giving with $87.5 \%$ response rate
(Figure 1). After randomisation, a total of 18 subjects in placebo group and 17 subjects in the intervention group were allocated. Demographic profiles of the subjects according to the supplementation group are presented

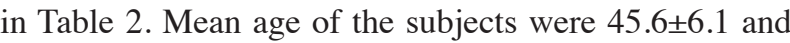
$44.7 \pm 4.6$ years, for placebo and P. minus, respectively. All of the subjects were Malays and both groups showed no significant difference with respect to socio-demographic profile. Anthropometric status which includes weight, height and BMI showed no significant difference between groups.

Table 3 shows the result of blood biomarkers changes from baseline after 6 weeks of supplementation in both group placebo and $P$. minus. Both placebo and $P$. minus group showed no significant difference in blood biomarkers at baseline. In the intervention group, there was a significant decrease $(p<0.05)$ of mean in uric acid, estimated glomerular filtration rate (eGFR), ratio total cholesterol to HDL and $\mathrm{HbA} 1 \mathrm{C}$ from baseline (303.2 \pm 71.3 $\mathrm{umol} / \mathrm{L}, 95.1 \pm 19.9 \mathrm{~mL} / \mathrm{min} / 1.73 \mathrm{~m}^{2}, 3.3 \pm 1.0 \mathrm{mmol} / \mathrm{L}$ and $5.7 \pm 0.4)$ to week $6(271.2 \pm 57.2 \mathrm{umol} / \mathrm{L}, 86.0 \pm 18.2 \mathrm{~mL} /$ $\mathrm{min} / 1.73 \mathrm{~m}^{2}, 3.2 \pm 0.7 \mathrm{mmol} / \mathrm{L}$ and $\left.5.4 \pm 0.3\right)$. The mean of

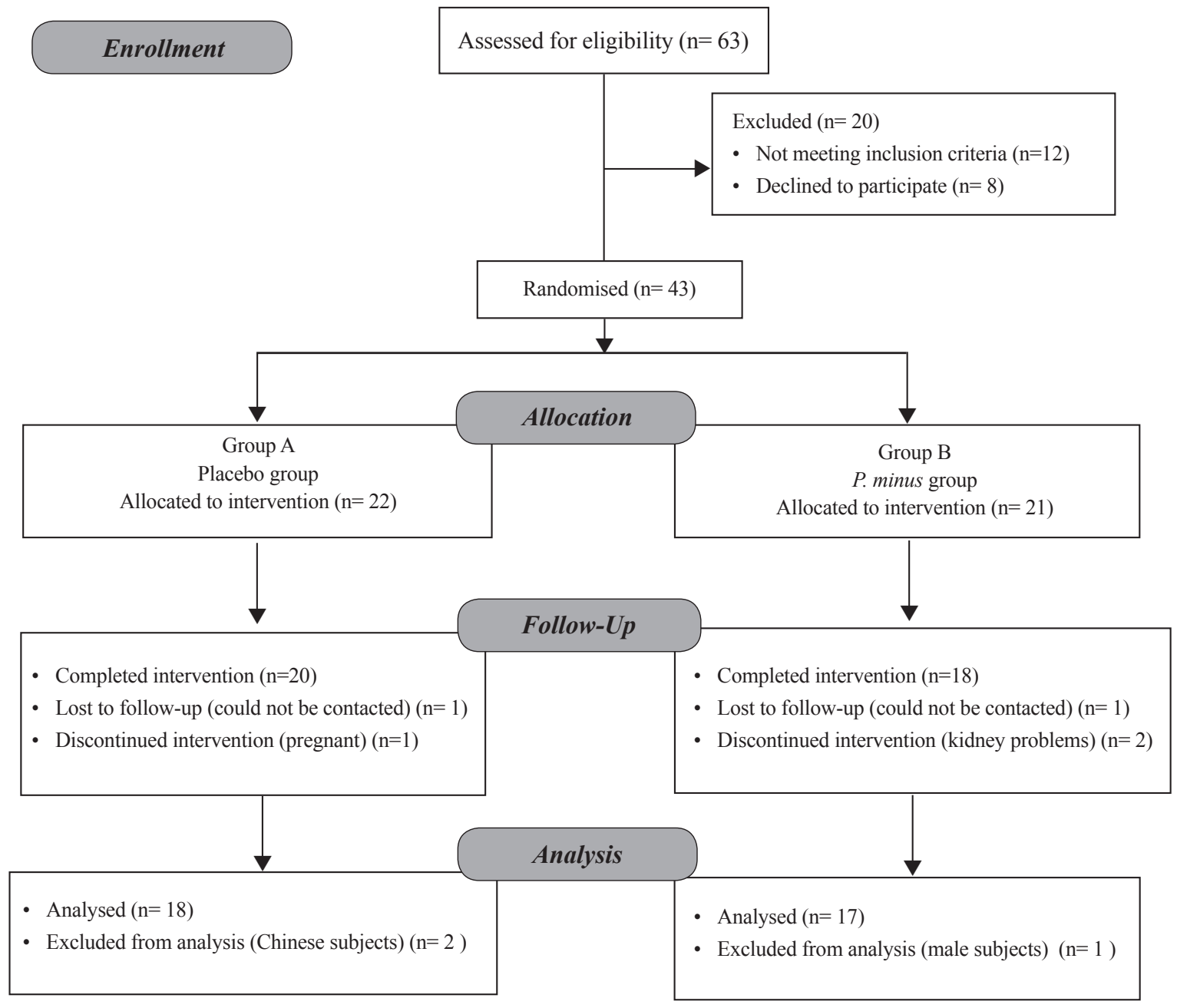

FIGURE 1. Flow diagram of study 
TABLE 2. Baseline characteristics of participants by intervention and placebo group

\begin{tabular}{|c|c|c|c|}
\hline Baseline characteristics & $\begin{array}{l}\text { Placebo } \\
(n=18)\end{array}$ & $\begin{array}{c}P . \text { minus } \\
(n=17)\end{array}$ & $\mathrm{P}$ value \\
\hline Mean age $(\text { year })^{1}$ & $45.6 \pm 6.1$ & $44.7 \pm 4.6$ & $0.623^{1}$ \\
\hline \multicolumn{4}{|l|}{ Marital status ${ }^{2}$} \\
\hline Married & $20(100.0 \%)$ & $17(94.1 \%)$ & $0.296^{2}$ \\
\hline Single & $0(0.0 \%)$ & $1(5.9 \%)$ & \\
\hline \multicolumn{4}{|l|}{ Level of education ${ }^{2}$} \\
\hline Secondary school & $8(44.4 \%)$ & $4(23.5 \%)$ & $0.362^{2}$ \\
\hline Certificate/Diploma & $2(11.1 \%)$ & $4(23.5 \%)$ & \\
\hline Degree & $8(44.4 \%)$ & $9(52.9 \%)$ & \\
\hline \multicolumn{4}{|l|}{ Occupation $^{2}$} \\
\hline Teacher & $8(44.4 \%)$ & $9(52.9 \%)$ & $0.615^{2}$ \\
\hline Non-teacher & $10(55.6 \%)$ & $8(47.1 \%)$ & \\
\hline Mean household income $(\mathrm{RM})^{1}$ & $7591.1 \pm 3904.2$ & $8250.6 \pm 5125.1$ & $0.670^{1}$ \\
\hline \multicolumn{4}{|l|}{ Anthropometric status ${ }^{1}$} \\
\hline Weight (kg) & $67.3 \pm 9.7$ & $66.9 \pm 11.7$ & $0.929^{1}$ \\
\hline Height $(\mathrm{cm})$ & $155.7 \pm 5.0$ & $154.3 \pm 5.5$ & $0.412^{1}$ \\
\hline BMI $\left(\mathrm{kg} / \mathrm{m}^{2}\right)$ & $27.7 \pm 3.9$ & $28.2 \pm 5.0$ & $0.734^{1}$ \\
\hline
\end{tabular}

${ }^{1}$ Continuous data were presented as mean \pm standard deviation, ${ }^{2}$ Categorical data were presented as number (\%), RM: Ringgit Malaysia

TABLE 3. Data on blood biomarkers at baseline and 6 weeks of intervention in control and intervention group (mean \pm SD)

\begin{tabular}{|c|c|c|c|c|}
\hline \multirow[b]{2}{*}{ Parameters } & \multicolumn{2}{|c|}{ Placebo $(n=18)$} & \multicolumn{2}{|c|}{ P. minus $(n=17)$} \\
\hline & $\begin{array}{c}\text { Baseline } \\
(\text { mean } \pm \mathrm{SD})\end{array}$ & $\begin{array}{c}\text { Week } 6 \\
(\text { mean } \pm \text { SD })\end{array}$ & $\begin{array}{c}\text { Baseline } \\
(\text { mean } \pm \mathrm{SD})\end{array}$ & $\begin{array}{c}\text { Week } 6 \\
(\text { mean } \pm \text { SD) }\end{array}$ \\
\hline \multicolumn{5}{|l|}{ Renal profil } \\
\hline Sodium (mmol/L) & $140.7 \pm 1.6$ & $142.7 \pm 1.9^{c}$ & $140.8 \pm 2.1$ & $141.5 \pm 2.6$ \\
\hline Potassium (mmol/L & $4.2 \pm 0.2$ & $4.5 \pm 0.5^{\mathrm{a}}$ & $4.3 \pm 0.4$ & $4.4 \pm 4.2$ \\
\hline Urea (mmol/L) & $3.7 \pm 1.1$ & $3.7 \pm 0.9$ & $3.7 \pm 0.8$ & $3.5 \pm 1.0$ \\
\hline Uric acid (umol/L) & $302.4 \pm 65.4$ & $290.7 \pm 69.5$ & $303.2 \pm 71.3$ & $271.2 \pm 57.2^{\mathrm{a}}$ \\
\hline Creatinine (umol/L) & $59.8 \pm 7.6$ & $65.1 \pm 8.8^{c}$ & $61.3 \pm 9.1$ & $66.6 \pm 10.0^{c}$ \\
\hline eGFR (mL/min/1.73m²) & $96.1 \pm 15.5$ & $87.0 \pm 13.0^{\mathrm{c}}$ & $95.1 \pm 19.9$ & $86.0 \pm 18.2^{\mathrm{c}}$ \\
\hline \multicolumn{5}{|l|}{ Liver function test } \\
\hline Total protein $(\mathrm{g} / \mathrm{L})$ & $78.1 \pm 3.4$ & $76.9 \pm 3.0^{\mathrm{a}}$ & $79.4 \pm 3.3$ & $77.6 \pm 4.6$ \\
\hline Albumin $(\mathrm{g} / \mathrm{L})$ & $44.8 \pm 2.1$ & $44.5 \pm 1.9$ & $44.9 \pm 2.9$ & $44.3 \pm 3.3$ \\
\hline Globulin $(\mathrm{g} / \mathrm{L})$ & $33.2 \pm 3.7$ & $32.5 \pm 2.9$ & $34.6 \pm 3.5$ & $33.5 \pm 4.5$ \\
\hline Bilirubin (umol/L) & $10.8 \pm 5.9$ & $10.4 \pm 6.5$ & $10.6 \pm 4.9$ & $9.2 \pm 3.4$ \\
\hline Alkaline phosphatase (U/L) & $73.9 \pm 20.2$ & $73.8 \pm 19.7$ & $72.6 \pm 14.7$ & $76.9 \pm 23.5$ \\
\hline GGT (U/L) & $27.0 \pm 21.1$ & $27.4 \pm 20.0$ & $28.5 \pm 12.9$ & $34.8 \pm 41.6$ \\
\hline Aspartate transferase (AST) (U/L) & $22.6 \pm 7.1$ & $24.0 \pm 11.4$ & $21.2 \pm 6.7$ & $32.4 \pm 30.4$ \\
\hline Alanine transaminase (ALT) (U/L) & $21.9 \pm 11.9$ & $19.7 \pm 9.8$ & $19.9 \pm 14.5$ & $33.3 \pm 40.6$ \\
\hline Lipid profile & $71 \pm 18.0$ & $74 \pm 17.5$ & $66 \pm 16.8$ & $72 \pm 14.9$ \\
\hline Total cholestrol $(\mathrm{mmol} / \mathrm{L})$ & $5.4 \pm 0.9$ & $5.4 \pm 0.9$ & $5.3 \pm 1.2$ & $5.4 \pm 1.3$ \\
\hline Triglycerides $(\mathrm{mmol} / \mathrm{L})$ & $0.9 \pm 0.4$ & $0.9 \pm 0.3$ & $0.9 \pm 0.2$ & $1.0 \pm 0.5$ \\
\hline $\mathrm{HDL}(\mathrm{mmol} / \mathrm{L})$ & $1.5 \pm 0.3$ & $1.5 \pm 0.3$ & $1.7 \pm 0.4$ & $1.7 \pm 0.4$ \\
\hline $\mathrm{LDL}(\mathrm{mmol} / \mathrm{L})$ & $3.5 \pm 0.9$ & $3.5 \pm 0.8$ & $3.2 \pm 1.0$ & $3.3 \pm 0.7$ \\
\hline Total cholestrol/HDL (mmol/L) & $3.7 \pm 1.1$ & $3.8 \pm 1.0$ & $3.3 \pm 1.0$ & $3.2 \pm 0.7^{\mathrm{a}}$ \\
\hline HbA1C (\%) & $5.9 \pm 0.8$ & $5.6 \pm 7.4^{b}$ & $5.7 \pm 0.4$ & $5.4 \pm 0.3^{c}$ \\
\hline
\end{tabular}

$* p<0.05$ mean difference between placebo and P. minus at baseline

$* * p<0.05$ mean changes between placebo and P. minus from baseline and week 6

${ }^{\mathrm{a}} p<0.05,{ }^{\mathrm{b}} p<0.01,{ }^{\mathrm{c}} p<0.001$

HDL: High density lipoprotein, LDL: Low density lipoprotein, HBA1c: Glycated hemoglobin 
creatinine increased significantly $(p<0.05)$ from baseline $(61.3 \pm 9.1 \mathrm{umol} / \mathrm{L})$ to week $6(66.6 \pm 10.0 \mathrm{umol} / \mathrm{L})$. In placebo group, mean of renal profile include sodium, potassium and creatinine had significantly increase from baseline $(140.7 \pm 1.6,4.2 \pm 0.2$ and $59.8 \pm 7.6 \mathrm{umol} / \mathrm{L})$ to week $6(142.7 \pm 1.9,4.5 \pm 0.5$ and $65.1 \pm 8.8 \mathrm{umol} / \mathrm{L})$. Meanwhile decreasing in mean was noted in eGFR and $\mathrm{HbA} 1 \mathrm{c}$ from baseline $\left(96.1 \pm 15.5 \mathrm{~mL} / \mathrm{min} / 1.73 \mathrm{~m}^{2}\right.$ and $\left.5.9 \pm 0.8\right)$ to week $6\left(87.0 \pm 13.0 \mathrm{~mL} / \mathrm{min} / 1.73 \mathrm{~m}^{2}\right.$ and $\left.5.6 \pm 7.4\right)$ in placebo group. However, no significant difference in mean changes between both groups after 6 weeks of intervention.

There was no significant difference in psychosocial status for both groups at baseline except for depression, fatigue, confusion and total mood disturbance. A significant decrease of mean POMS (tension, depression, anger and TMD (Total Mood Disturbance)) were observed in the intervention group from baseline $(10.9 \pm 4.5,11.8 \pm 6.4$, $11.7 \pm 4.9$ and $32.0 \pm 21.8)$ to week $6(8.6 \pm 5.0,8.1 \pm 8.2$, $8.7 \pm 6.1$ and $21.1 \pm 25.1)$ with $p$ value of $0.038,0.019$, 0.011 and 0.010 , respectively (Table 4). Mean changes in POMS (anger) was significantly difference in $P$. minus group compared to placebo from baseline to week 6 . Furthermore, mean of quality of life (energy/fatigue, social functioning and general health) in the intervention group have significantly increased from baseline (62.4 \pm 15.9 ,
$79.4 \pm 19.7,65.6 \pm 16.8)$ to week $6(68.2 \pm 13.7,91.9 \pm 8.8$ and $71.8 \pm 14.1)$ with $p$ value of $0.022,0.008$ and 0.028 , respectively. There were no significant difference in both parameters of mood and quality of life in placebo group.

Improvement in the mean score of digit span, CTMT (Trail 1, Trail 2, Trail 5 and Composite Index), WASI (IQ performance and IQ full) and CNSVS (cognitive flexibility, psychomotor speed and executive function) from baseline to week 6 was found for both groups (Table 5). However, only CTMT (Trail 3) shows significantly difference in mean changes between treatment and placebo group at week 3 and baseline. In addition, CTMT (Trail 5) shows a significant difference in mean changes between both groups at baseline and week 6. Mean score for digit span improved from baseline to week $6(p=0.030)$ in $P$. minus group and in placebo group $(p=0.005)$. Both groups showed an increment in the mean score for CTMT composite index for baseline and week 6 . In addition, P. minus exert highly significant difference $(p=0.001)$ as compared to the placebo group $(p<0.05)$ from baseline to week 6 in the Full Scale IQ of WASI. Similar trend was observed for executive function in CNSVS which shown higher significant level in P. minus group as compared to placebo group. Besides, significant improvement in cognitive flexibility domain in CNSVS was seen from week 3 for $P$. minus group, but was only

TABLE 4. Data on psychosocial status at baseline, 3 weeks and 6 weeks of intervention in control (mean \pm SD) and intervention group (mean \pm SD)

\begin{tabular}{|c|c|c|c|c|c|c|}
\hline \multirow[b]{2}{*}{ Parameters } & \multicolumn{2}{|c|}{ Baseline (mean $\pm \mathrm{SD}$ ) } & \multicolumn{2}{|c|}{ Week $3($ mean $\pm \mathrm{SD})$} & \multicolumn{2}{|c|}{ Week $6($ mean \pm SD $)$} \\
\hline & $\begin{array}{l}\text { Placebo } \\
(n=18)\end{array}$ & $\begin{array}{l}\text { P. minus } \\
(n=17)\end{array}$ & $\begin{array}{l}\text { Placebo } \\
(n=18)\end{array}$ & $\begin{array}{l}\text { P. minus } \\
(n=17)\end{array}$ & $\begin{array}{l}\text { Placebo } \\
(n=18)\end{array}$ & $\begin{array}{l}\text { P. minus } \\
(n=17)\end{array}$ \\
\hline \multicolumn{7}{|l|}{ POMS } \\
\hline Tension & $8.3 \pm 3.8$ & $10.9 \pm 4.5$ & $9.7 \pm 6.0$ & $8.6 \pm 5.6$ & $8.4 \pm 5.4$ & $8.6 \pm 5.0^{\mathrm{c}}$ \\
\hline Depression & $5.3 \pm 4.7$ & $11.8 \pm 6.4^{*}$ & $9.2 \pm 10.4$ & $7.9 \pm 9.4$ & $7.2 \pm 10.5$ & $8.1 \pm 8.2^{\mathrm{c}}$ \\
\hline Anger & $8.3 \pm 5.4$ & $11.7 \pm 4.9$ & $9.3 \pm 7.7$ & $9.1 \pm 6.5$ & $8.9 \pm 5.4$ & $8.7 \pm 6.1 * * * c$ \\
\hline Vigour & $19.6 \pm 7.1$ & $19.2 \pm 5.2$ & $18.8 \pm 5.1$ & $17.7 \pm 5.3$ & $19.7 \pm 5.7$ & $18.6 \pm 4.6$ \\
\hline Fatigue & $6.2 \pm 3.4$ & $9.1 \pm 4.2 *$ & $7.7 \pm 5.6$ & $7.4 \pm 5.3$ & $6.4 \pm 5.6$ & $7.3 \pm 4.6$ \\
\hline Confusion & $5.6 \pm 2.3$ & $7.6 \pm 2.6^{*}$ & $7.3 \pm 4.0$ & $6.7 \pm 3.2$ & $6.7 \pm 4.0$ & $6.6 \pm 3.2$ \\
\hline $\begin{array}{l}\text { Total mood } \\
\text { disturbance }\end{array}$ & $14.2 \pm 16.2$ & $32.0 \pm 21.8^{*}$ & $24.8 \pm 29.7$ & $21.2 \pm 30.9$ & $18.1 \pm 29.4$ & $21.1 \pm 25.1^{\mathrm{c}}$ \\
\hline \multicolumn{7}{|l|}{ SF-36 } \\
\hline $\begin{array}{l}\text { Physical } \\
\text { Functioning }\end{array}$ & $90.0 \pm 11.4$ & $82.6 \pm 17.6$ & $89.2 \pm 11.9$ & $86.8 \pm 12.6$ & $91.7 \pm 9.7$ & $86.9 \pm 13.3$ \\
\hline $\begin{array}{l}\text { Role limitation due } \\
\text { to physical health }\end{array}$ & $75.0 \pm 35.4$ & $79.4 \pm 29.6$ & $73.6 \pm 34.8$ & $75.0 \pm 35.4$ & $72.2 \pm 39.2$ & $88.2 \pm 33.2$ \\
\hline $\begin{array}{l}\text { Role limitation due to } \\
\text { emotional problems }\end{array}$ & $88.9 \pm 28.0$ & $78.4 \pm 37.2$ & $98.1 \pm 7.7$ & $82.4 \pm 31.4$ & $90.7 \pm 27.5$ & $92.2 \pm 22.1$ \\
\hline Energy/fatigue & $67.2 \pm 14.8$ & $62.4 \pm 15.9$ & $72.8 \pm 14.6$ & $69.4 \pm 13.7^{\mathrm{a}}$ & $70.3 \pm 19.0$ & $68.2 \pm 13.7^{\mathrm{c}}$ \\
\hline Emotional wellbeing & $78.0 \pm 10.5$ & $73.2 \pm 13.7$ & $82.4 \pm 9.1$ & $77.9 \pm 13.4$ & $77.3 \pm 17.5$ & $76.0 \pm 12.6$ \\
\hline Social functioning & $84.0 \pm 19.6$ & $79.4 \pm 19.7$ & $88.9 \pm 15.4$ & $90.4 \pm 10.4^{\mathrm{a}}$ & $85.4 \pm 15.0$ & $91.9 \pm 8.8^{\mathrm{c}}$ \\
\hline Bodily pain & $79.0 \pm 18.9$ & $69.9 \pm 17.8$ & $83.6 \pm 14.0$ & $78.4 \pm 15.1$ & $83.2 \pm 19.8$ & $73.8 \pm 10.1$ \\
\hline General health & $70.6 \pm 18.0$ & $65.6 \pm 16.8$ & $74.4 \pm 17.5$ & $72.1 \pm 14.9$ & $71.7 \pm 20.3$ & $71.8 \pm 14.1^{\mathrm{c}}$ \\
\hline
\end{tabular}

$* p<0.05$ mean difference between placebo and $P$. minus at baseline

** $p<0.05$ mean changes between placebo and P. minus from baseline and week 3 , $* * * p<0.05$ mean changes between placebo and $P$. minus from baseline and week 6 ${ }^{\mathrm{a}} p<0.05$ at baseline and week $3,{ }^{\mathrm{b}} p<0.05$ at Week 3 and Week $6,{ }^{,} p<0.05$ at baseline and Week 6

POMS: Profile of Mood States, SF-36: 36-Item Short form Health Survey 
TABLE 5. Data on cognition at baseline, 3 weeks and 6 weeks of intervention in control $($ mean $\pm \mathrm{SD})$ and intervention group (mean $\pm \mathrm{SD})$

\begin{tabular}{|c|c|c|c|c|c|c|}
\hline \multirow[b]{2}{*}{ Parameters } & \multicolumn{2}{|c|}{ Baseline $($ mean $\pm \mathrm{SD})$} & \multicolumn{2}{|c|}{ Week $3($ mean \pm SD) } & \multicolumn{2}{|c|}{ Week $6($ mean \pm SD $)$} \\
\hline & $\begin{array}{c}\text { Placebo } \\
(n=18)\end{array}$ & $\begin{array}{l}\text { P. minus } \\
(n=17)\end{array}$ & $\begin{array}{c}\text { Placebo } \\
(n=18)\end{array}$ & $\begin{array}{l}\text { P. minus } \\
(n=17)\end{array}$ & $\begin{array}{c}\text { Placebo } \\
(n=18)\end{array}$ & $\begin{array}{l}\text { P. minus } \\
(n=17)\end{array}$ \\
\hline Digit span & $8.2 \pm 1.8$ & $8.8 \pm 2.5$ & $10.1 \pm 3.3^{\mathrm{a}}$ & $9.5 \pm 1.5$ & $9.8 \pm 2.5^{\mathrm{c}}$ & $9.8 \pm 2.0^{\mathrm{c}}$ \\
\hline \multicolumn{7}{|l|}{ RAVLT } \\
\hline Learning & $48.7 \pm 10.4$ & $51.8 \pm 9.3$ & $50.2 \pm 12.1$ & $50.4 \pm 6.1$ & $49.1 \pm 10.7$ & $51.2 \pm 7.9$ \\
\hline Recall & $50.1 \pm 9.9$ & $51.4 \pm 7.4$ & $50.4 \pm 10.1$ & $49.4 \pm 9.6$ & $49.0 \pm 10.3$ & $51.0 \pm 7.5$ \\
\hline \multicolumn{7}{|l|}{ CTMT } \\
\hline Trail 1 & $38.8 \pm 9.5$ & $40.2 \pm 7.0$ & $48.6 \pm 7.0^{\mathrm{a}^{*}}$ & $47.6 \pm 6.3^{\mathrm{a}^{*}}$ & $52.0 \pm 10.0^{\mathrm{b}, \mathrm{c}^{*}}$ & $49.8 \pm 8.1^{\mathrm{c}^{*}}$ \\
\hline Trail 2 & $44.8 \pm 8.7$ & $43.6 \pm 7.9$ & $51.7 \pm 11.3^{\mathrm{a}}$ & $42.9 \pm 5.8$ & $52.1 \pm 11.8^{\mathrm{b}, \mathrm{c}}$ & $49.6 \pm 11.5^{\mathrm{c}}$ \\
\hline Trail 3 & $41.6 \pm 10.2$ & $42.6 \pm 9.5$ & $46.6 \pm 10.0^{\mathrm{a}}$ & $47.2 \pm 10.2 * * a$ & $49.5 \pm 10.4^{c}$ & $46.1 \pm 7.3$ \\
\hline Trail 4 & $41.2 \pm 9.5$ & $39.1 \pm 6.0$ & $43.4 \pm 11.0$ & $42.9 \pm 7.8$ & $44.1 \pm 8.7$ & $45.5 \pm 7.9^{\mathrm{c}}$ \\
\hline Trail 5 & $42.0 \pm 8.2$ & $44.1 \pm 5.6$ & $47.1 \pm 10.2^{\mathrm{a}}$ & $44.8 \pm 7.6$ & $50.9 \pm 8.0^{\mathrm{b}, \mathrm{c}^{*}}$ & $48.3 \pm 7.5^{* * * c}$ \\
\hline Composite index & $40.6 \pm 8.0$ & $40.8 \pm 6.6$ & $46.7 \pm 8.7^{\mathrm{a}}$ & $44.2 \pm 6.4^{\mathrm{a}}$ & $49.2 \pm 9.1^{\mathrm{b}, \mathrm{c}^{*}}$ & $47.2 \pm 7.0^{\mathrm{c}^{*}}$ \\
\hline \multicolumn{7}{|l|}{ WASI } \\
\hline IQ verbal & $109.7 \pm 11.6$ & $108.5 \pm 10.3$ & $108.5 \pm 6.3$ & $107.2 \pm 7.0$ & $109.1 \pm 5.8$ & $108.8 \pm 5.7$ \\
\hline IQ performance & $111.3 \pm 10.1$ & $107.1 \pm 11.8$ & $116.4 \pm 10.5^{\mathrm{a}}$ & $114.9 \pm 6.9^{\mathrm{a}}$ & $117.8 \pm 6.0^{\mathrm{b}, \mathrm{c}}$ & $119.2 \pm 8.4 * * * c^{*}$ \\
\hline IQ full & $111.6 \pm 9.3$ & $108.7 \pm 10.0$ & $114.2 \pm 8.2$ & $112.2 \pm 6.6$ & $114.5 \pm 5.0^{c}$ & $115.6 \pm 6.0^{\mathrm{c}}$ \\
\hline \multicolumn{7}{|l|}{ CNS Vital Sign } \\
\hline Visual memory & $83.7 \pm 18.6$ & $87.8 \pm 12.7$ & $80.2 \pm 14.5$ & $88.3 \pm 13.2$ & $83.0 \pm 16.3$ & $85.8 \pm 16.3$ \\
\hline Psychomotor speed & $89.1 \pm 22.0$ & $99.3 \pm 13.7$ & $90.3 \pm 16.6$ & $98.2 \pm 14.4$ & $95.2 \pm 16.6$ & $103.2 \pm 16.6^{\mathrm{b}}$ \\
\hline Reaction time & $92.3 \pm 16.3$ & $84.1 \pm 17.8$ & $95.3 \pm 15.3$ & $90.9 \pm 14.3^{\mathrm{a}}$ & $97.6 \pm 11.9$ & $90.6 \pm 11.9$ \\
\hline Cognitive flexibility & $97.2 \pm 14.9$ & $94.1 \pm 11.9$ & $104.1 \pm 15.9$ & $101.9 \pm 12.7^{\mathrm{a}}$ & $108.0 \pm 11.3^{\mathrm{c}}$ & $107.0 \pm 11.3^{\mathrm{c}^{*}}$ \\
\hline Processing speed & $101.5 \pm 15.1$ & $102.6 \pm 14.4$ & $106.4 \pm 15.5^{\mathrm{a}}$ & $107.0 \pm 17.2$ & $109.6 \pm 24.5^{\mathrm{c}}$ & $114.8 \pm 24.5^{\mathrm{b}, \mathrm{c}}$ \\
\hline Executive function & $100.1 \pm 14.2$ & $95.2 \pm 11.4$ & $105.3 \pm 13.3$ & $102.4 \pm 12.9^{\mathrm{a}}$ & $108.2 \pm 11.7^{\mathrm{c}}$ & $107.0 \pm 11.7^{\mathrm{c}^{*}}$ \\
\hline Motor speed & $85.2 \pm 25.1$ & $97.0 \pm 14.3$ & $83.3 \pm 18.3$ & $93.4 \pm 15.9$ & $87.5 \pm 13.2$ & $94.5 \pm 13.2$ \\
\hline
\end{tabular}

$* p<0.05$ mean difference between placebo and P. minus at baseline

${ }^{* *} p<0.05$ mean changes between placebo and P. minus from baseline and week 3 , *** $p<0.05$ mean changes between placebo and $P$. minus from baseline and week 6 ${ }^{a} p<0.05$ at baseline and Week $3,{ }^{a *} p<0.01$ at baseline and Week $3,{ }^{b} p<0.05$ at Week 3 and Week $6, p<0.05$ at baseline and Week $6,{ }^{*} p<0.01$ at baseline and Week 6 RAVLT: Rey Auditory Verbal Learning Test, CTMT: Comprehensive trail making test, WASI: Wechsler Abbreviated Scale of Intelligence, CNS Vital Sign: Central Nervous System Vital Sign

seen at week 6 for placebo group. No adverse effects were reported by the subjects from both groups throughout the study period.

\section{DISCUSSION}

To date, most of previous studies have focused on assessing the efficacy of $P$. minus on animal (George et al. 2014; Ming et al. 2013; Norliza et al. 2013). Hence, there are a limited number of studies reporting on the efficacy of $P$. minus extract supplementation on cognitive function, mood status and quality of life in human. In this present study, 6 week supplementation of $P$. minus extract among healthy women aged 35 to 55 years was found to improve some domains in mood state and quality of life. Significant improvements were noted in POMS for tension, depression, anger and total mood disturbance and SF-36 domain including energy/fatigue, social functioning and general health in P. minus group. With respect to quality of life assessed by SF-36, the significant improvement was seen from week 3 onward for energy/fatigue and social functioning domain. Whereas, there was no improvement seen in the placebo group for mood and quality of life until week 6 of supplementation.

Several studies have reported that leaves of $P$. minus are high in phytochemicals particularly flavonoid which associated with their antioxidant activity (Maizura et al. 2011; Urones et al. 1990). Flavonoid possessed the ability to delay or prevent degenerative diseases caused by oxidative damage, thus facilitate in the reduction of oxidative stress and lipid peroxidation in neuronal membranes (Spencer 2009). To exert the effect on cognition or mood, flavonoids must arrive at the central nervous system (CNS) through the blood brain barrier (Jäger \& Saaby 2011). Studies have shown that quercetin-3-glucuronide can be absorbed through the small intestine and reach the central nervous system (CNS) (Lee et al. 2001). In a cell culture study, quercetin extracted from onion has been reported to permeate across the blood brain barrier and has the ability to impede the neuron cell death by hydrogen peroxide (Dan et al. 2011). In an animal study, oral administration of quercetin has shown to reduce the increment of oxidative 
stress in the brain thus proved the ability of this compound to permeate to the brain (Ishisaka et al. 2011). Although in the present study we have not measure the bioavailability of quercetin from P. minus, previous supplementation studies using quercetin have reported the increase in plasma level of quercetin after two week up to 12 weeks administration (Egert et al. 2008; Jin et al. 2010).

Quercetin and quercetin-3-glucuronide were reported to have an impact on gamma-aminobutyric acid (GABA) receptors producing sedation, anxiolytic or anticonvulsive effects (Jäger \& Saaby 2011). In addition, structural formula of $P$. minus extract which consist of two flavonoids namely quercetin-3-glucoronide and quercitrin has been reported to possess anti-depression property (Pathak et al. 2013). Thus, these components found in P. minus extract might explain its ability in improving mood state particularly in tension, depression, anger and total mood disturbance and domain in SF-36 (energy/fatigue, social functioning and general health). Similarly, Udani (2013) has observed a significant improvement in tension, depression and anger of POMS after the supplementation of SuperUlam, a mixture of natural herbs including P. minus. The synergistic effect mixture of vitamins and minerals in $P$. minus capsule also might potentially affect the mood parameters. Previous study among elderly has reported that supplementation of multivitamin formulation consist of vitamins, minerals, antioxidants and herbal extracts has improved alertness and reducing negative mood symptom in the intervention group (Harris et al. 2011). Study by Tildesley et al. (2005) has suggested an improvement in mood after acute administration of Salvia lavandulaefolia in the form of capsule. Supplementation of Nigella sativa L. or also known as black cumin for four weeks has shown to improve mood and decrease anxiety through their potent antioxidant and anticholinesterase activities of the herbs (Sayeed et al. 2014). On the contrary, Panax ginseng has shown no effect on total mood disturbance after 8 weeks supplementation with different dosages (Cardinal \& Engels 2001). The effect of herbal supplementation on mood can vary according to dosage, duration and the forms of supplementation given to the subjects.

In the present study, both P. minus and control group have shown an improvement in cognitive test administered. One of the possible explanations is due to practice effect. This term has been defined as the improvements in cognitive performance caused by practicing a task or repetition of the cognitive battery (Heiman 2002). Participants were required to repeat the same version of cognitive tests during baseline, week 3 and week 6 , thus, this might exposed them to practice effect and led to an improvement in cognitive performance. In the present study, only computerized CNSVS had different test versions. Study by Collie et al. (2003) has reported a significant improvement due to practice effect after first and second administration of cognitive test battery to the subjects. However, the following administration did not show any improvement in the test performance. Although practice effect has been linked as a source of error, earlier study has suggested that it can be a beneficial tool to monitor cognitive status specifically among patients with neurodegenerative disorders at earlier stage (Duff et al. 2007).

There are some strengths in this study that enhance confident in the results obtained, including the use of randomised, double-blind, placebo controlled methods, a full 6 weeks supplementation, blood monitoring at baseline and week 6 of the study and multiple assessments of cognition test. However, this study is limited by a short duration of supplementation. In addition, only one male subject has successfully completed the study and the data was excluded from the analysis, thus no comparison between genders can be made. This research will serve as a base for future studies to investigate the benefits of P. minus supplementation on cognitive and mood in a wide range of population which include different genders and races. Moreover, it would be interesting to assess the mechanism of action of P. minus specifically on mood.

\section{CONCLUSION}

In conclusion, this study suggested that 6 weeks supplementation of $P$. minus (LineMinus ${ }^{\mathrm{TM}}$ ) had significantly improved mood specifically in tension, depression, anger and total mood disturbance. Similarly, the same trend was found for quality of life components including energy/fatigue, social functioning and general health. On the other hand, improvements in cognitive functions were observed in both intervention and control group. Further investigations in determining the effect of $P$. minus at cellular and molecular level are strongly recommended.

\section{ACKNOWLEDGMENTS}

Financial support for the present study was provided by Biotropics Malaysia Berhad (Selangor, Malaysia). We thanked all participated schools and institution who gave their utmost cooperation in completing the study. We also would like to thank all the field workers during data collection period. We declare that the study was sponsored by Biotropics Malaysia Berhad (Selangor, Malaysia).

\section{REFERENCES}

Al-Qari, A. 2006. Ensiklopedia Herba-herba Sekeliling Anda. Selangor: Percetakan Zafar Sdn Bhd.

Azlim Almey, A.A., Ahmed Jalal Khan, C., Syed Zahir, I., Mustapha Suleiman, K., 'Aisyah, M.R. \& Kamarul Rahim, K. 2010. Total phenolic content and primary antioxidant activity of methanolic and ethanolic extracts of aromatic plants' leaves. Intern. Food Res. J. 17: 1077-1084.

Brazier, J., Harper, R., Jones, N.M., O' cathain, A., Thomas, K.J., Usherwood, T. \& Westlake, L. 1992. Validating the SF-36 health survey questionnaire: New outcome measure for primary care. Br. Med.J. 305: 160-164.

Cardinal, B.J. \& Engels, H.J. 2001. Ginseng does not enhance psychological well-being in healthy, young adults: Results 
of a double-blind, placebo-controlled, randomised clinical trial. J. Am. Diet. Assoc. 101: 655-660.

Cheah, K., Noorzaleha, S., Lee, H., Son, R., Sabrina, S. \& Sim, J. 2008. Comparison of PCR finger printing techniques for the discrimination of Salmonella enteric subsp. enteric Serovarweltevreden isolated from indigenous vegetables in Malaysia. World J. Microb. Biot. 24: 327-335.

Ching,L.S. \& Mohamed, S. 2001. Alpha-tocopherol content in 62 edible tropical plants. J. Agric. Food Chem. 49: 3101-3105.

Collie, A., Maruff, P., Darby, D.G. \& McStephen, M. 2003. The effects of practice on the cognitive test performance of neurologically normal individuals assessed at brief test-retest intervals. J. Int. Neuropsychol. Soc. 9: 419-428.

Dan, H., Du, W.T. \& Fan, X.J. 2011. Study of flavanoids extracted from onion on the blood-brain barrier permeation and neuroprotective effects. Zhongguo Zhong Xi Yi Jie He Za Zhi 31: 1674-1679.

Duff, K., Beglinger, L.J., Schultz, S.K., Moser, D.J., McCaffrey, R.J., Haase, R.F., Westervelt, H.J., Langbehn, D.R. \& Paulsen, J.S. 2007. Practice effects in the prediction of longterm cognitive outcome in three patient samples: A novel prognostic index. Arch. Clin. Neuropsychol. 22: 15-24.

Egert, S., Wolffam, S., Bosy-Westphal, A., Boesch-Saadatmandi, C., Wagner, A.E., Frank, J., Rimbach, G. \& Mueller, M.J. 2008. Daily quercetin supplementation dose-dependently increases plasma quercetin concentrations in healthy humans. J. Nutr. 138(9): 1615-1621.

Garcia-Garcia, P., Lopez-Munoz, F., Rubio, G., Martin-Agueda, B. \& Alamo, C. 2008. Phytotherapy and psychiatry: Bibliometric study of the scientific literature from the last 20 years. Phytomedicine 15: 566-576.

George, A., Ng, C.P., O’Callaghan, M., Jensen, G.S. \& Wong, H.J. 2014. In vitro and ex-vivo cellular antioxidant protection and cognitive enhancing effects of an extract of Polygonum minus Huds (Lineminus ${ }^{\mathrm{TM}}$ ) demonstrated in a Barnes Maze animal model for memory and learning. BMC Complement Altern. Med. doi: 10.1186/1472-6882-14-161.

Groth-Marnat, G.E. 2000. Neuropsychological Assessment in Clinical Practice: A Guide to Test Interpretation and Integration. New Jersey: John Wiley \& Sons Inc. pp. 129-194.

Gualtieri, C.T. \& Johnson, L.G. 2006. Reliability and validity of a computerized neurocognitive test battery, CNS Vital Signs. Arch. Clin. Neuropsychol. 21: 623-643.

Harris, E., Kirk, J., Rowsell, R., Vitetta, L., Sali, A., Scholey, A.B. \& Pipingas, A. 2011. The effect of multivitamin supplementation on mood and stress in healthy older men. Hum. Psychopharmacol. 26: 560-567.

Heiman, G.W. 2002. Research Methods in Psychology. 3rd ed. Boston \& New York: Houghton Mifflin Company.

Herbal Medicine Research Centre, Institute for Medical Research. 2002. Compendium of medicinal plants used in Malaysia. Vol. 1. Kuala Lumpur: Institute for Medical Research.

Huda-Faujan, N., Noriham, A., Norrakiah, S. \& Babji, S. 2009. Antioxidant activity of plants methanolic extracts containing phenolic compounds. Afric. J. Biotech. 8: 484-489.

Ishisaka, A., Ichikawa, S., Sakakibara, H., Piskula, M.K., Nakamura, T., Kato, Y., Ito, M., Miyamoto, K., Tsuji, A., Kawai, Y. \& Terao, J. 2011. Accumulation of orally administered quercetin in brain tissue and its antioxidative effects in rats. Free Radic. Biol. Med. 51: 1329-1336.

Jäger,A.K. \& Saaby,L. 2011. Flavonoids and the CNS. Molecules 16: $1471-1485$.
Jin, F., Nieman, D.C., Shanely, R.A., Knab, A.M., Austin, M.D \& Sha, W. 2010. The variable plasma quercetin response to 12-week quercetin supplementation in humans. Eur.J. Clin. Nutr. 64(7): 692-697.

Joseph, S., Sugumaran, M. \& Kate, L.W.L. 2005. Herbs of Malaysia: An Introduction to the Medicinal, Culinary, Aromatic and Cosmetic Use of Herbs. Selangor: Federal Publications Sdn. Bhd.

Kinrys, G., Coleman, E. \& Rothstein, E. 2009. Natural remedies for anxiety disorders: Potential use and clinical applications. Depress Anxiety 26: 259-265.

Lakhan, S.E., \& Viera, K.F. 2010. Nutritional and herbal supplements for anxiety and anxiety-related disorders: Systematic review. Nutr. J. 9: 1-14.

Lee, M.H., Lin, R.D., Shen, L.Y., Yang, L.L., Yen, K.Y. \& Hou, W.C. 2001. Monamine oxidase B and free radical scavenging activities of natural flavonoids in Melastoma candidum D. Don. J. Agric. Food Chem. 4: 5551-5555.

Maizura, M.,Aminah, A. \& Wan Aida, W.M. 2011. Total phenolic content and antioxidant activity of kesum (Polygonum minus), ginger (Zingiber officinale) and turmeric (Curcuma longa) extract. Int. Food Res. J. 18: 529-534.

McNair, D.M., Lorr, M. \& Droppelman, L.F. 1971. Manual for the Profile of Mood States. San Diego, California: Educational and Industrial Testing Service.

Ming, Y.K., Zulkawi, N.B., Vandana Kotak, C. \& Choudhary, Y.K. 2013. Acute and sub-acute oral toxicity of Polygonum minus aqueous extract (Biotropics ${ }^{\circledR}$ Pm101) in Wistar rats. Int. J. Pharm Pharm. Sci. 5: 120-124.

Narasimhulu, G. \& Mohamed, J. 2014. Medicinal phytochemical and pharmacological properties of kesum (Polygonum minus linn.): A mini review. Int. J. Pharm Pharm. Sci. 6(4): 1-7.

Norliza, M., Elliza, M., Yap, C.S., Nor Syahira, S., Amri, D., Ahmad Fadhil, A., Norazlina, M., Ahmad Nazrun, S., Abdul Salam, B. \& Ima Nirwana, S. 2013. Acute and subacute toxicity of Persicaria minor in Wistar rats. Asian J. Anim. Sci. 7: 47-55.

Pathak, L., Agrawal, Y. \& Dhir, A. 2013. Natural polyphenols in the management of major depression. Expert Opin. Investig. Drugs 22: 863-880

Qader, S.W., Abdulla, M.A., Chua, L.S. \& Hamdan, S. 2012. Potential bioactive property of Polygonum minus Huds (kesum) - Review. Scientific Research and Essays 7: 90-93.

Qader, S.W., Abdulla, M.A., Chua, L.S., Najim, N., Zain, M.M. \& Hamdan, S. 2011. Antioxidant, total phenolic content and cytotoxicity evaluation of selected Malaysian plants. Molecules 16: 3433-3443.

Rey, A. 1964. L'Examen Clinique enPsychologie. Paris: Press Universitaire de France.

Reynolds, C.R. 2002. Comprehensive Trail Making Test (CTMT). Austin: Pro-Ed.

Saeed, S.A., Bloch, R.M. \& Antonacci, D.J. 2007. Herbal and dietary supplements for treatment of anxiety disorders. Am. Fam. Physician 76: 549-556.

Sayeed, M.S., Shams, T., Hossain, S.F., Rahman, M.R., Mostofa, A.G.M., Kadir, M.F., Mahmood, S. \& Asaduzzaman, M. 2014. Nigella sativa L. seeds modulate mood, anxiety and cognition in healthy adolescent males. J. Ethnopharmacol. 152: $156-162$.

Scholey, A., Ossoukhova, A., Owen, L., Ibbara, A., Pipingas, A., He, K., Roller, M. \& Stough, C. 2010. Effects of American ginseng (Panax quinquefolius) on neurocognitive function: An acute, randomised, double-blind, placebo-controlled, crossover study. Psychopharmacology (Berl.) 212: 345-356. 
Silberstein, R.B., Pipingas, A., Song, J., Camfield, D.A., Nathan, P.J.\& Stough, C. 2011. Examining brain-cognition effects of ginkgo biloba extract: Brain activation in the left temporal and left prefrontal cortex in an object working memory task. Evid. Based Complementary Altern. Med. http://dx.doi. org/10.1155/2011/164139.

Spencer, J.P. 2009. Flavonoids and brain health: Multiple effects underpinned by common mechanisms. Genes Nutr. 4: 243250 .

Sumazian, Y., Ahmad, S., Mansor, H. \& Mahmood, M. 2010, Antioxidant activities, flavonoids, ascorbic acid and phenolic content of Malaysian vegetables. J. Med. Plants Res. 4: 881-890.

Suzana, S., Ainor, F.A., Arina, S.N.I., Hanis Mastura, Y., Normah, C.D., Zahara, A.M. \& Manal, M.B. 2015. The effect of Polygonum minus extract on cognitive and psychological parameters according to mood status among middle-aged women: A randomised, double-blind, placebo-controlled study. Clin. Interv. Aging 10: 1505-1520.

Tanaka, T. 1976. Tanaka's Cyclopedia of Edible Plants of the World. Tokyo, Japan: Keigaku Publishing Co. p. 574.

Tildesley, N., Kennedy, D.O., Perry, E.K., Ballard, C.G., Wesnes, K.A. \& Scholey, A.B. 2005. Positive modulation of mood and cognitive performance following administration of acute doses of Salvia lavandulaefolia essential oil to healthy young volunteers. Physiol. Behav. 83: 699-709.

Udani, J.K. 2013. Effects of SuperUlam on supporting concentration and mood: A randomised, double-blind, placebocontrolled crossover study. Evid. Based Complementary Altern. Med. doi.org/10.1155/2013/238454.

Urones, G., Sanche, I., Gomez, B.\& Basabe, P. 1990. Flavonoids from Polygonum minus. Phytochem. 29: 3687-3689.

Wechsler, D. 1999. Wechsler Abbreviated Scale of Intelligence. The Psychological Corporation: Harcourt Brace \& Company, New York.
World Health Organization, WHO. 2004. Promoting mental health: concepts, emerging evidence, practice. http://www. who.intlmentalhealth/evidence/en/promoting mhh.pdf. Accessed 25 May 2015.

Hanis Mastura Yahya*, Siti Nur Arina Ismail \&

Ainor Farahin Aziz

Nutritional Sciences Programme

School of Healthcare Sciences, Faculty of Health Sciences

Universiti Kebangsaan Malaysia, Jalan Raja Muda Abd Aziz 50300 Kuala Lumpur, Wilayah Persekutuan Malaysia

Suzana Shahar \& Bibi Nabihah Abdul Hakim

Dietetics Programme

School of Healthcare Sciences, Faculty of Health Sciences Universiti Kebangsaan Malaysia, Jalan Raja Muda Abd Aziz 50300 Kuala Lumpur, Wilayah Persekutuan

Malaysia

Normah Che Din

Health Psychology Programme

School of Healthcare Sciences, Faculty of Health Sciences Universiti Kebangsaan Malaysia, Jalan Raja Muda Abd Aziz 50300 Kuala Lumpur, Wilayah Persekutuan

Malaysia

*Corresponding author; email: hanis.yahya@ukm.edu.my

Received: 4 December 2015

Accepted: 8 June 2016 